\title{
DIAGNOSING ANGINA USING A SIMPLE NEURAL NETWORK ARCHITECTURE
}

\author{
Bulgiba AM \\ Department of Social and Preventive Medicine, Faculty of Medicine, University of Malaya, 50603 Kuala Lumpur, Malaysia
}

\begin{abstract}
The aim of the study was to research the use of a simple neural network in diagnosing angina in patients complaining of chest pain. A total of 887 records were extracted from the electronic medical record system (EMR) in Selayang Hospital, Malaysia. Simple neural networks (simple perceptrons) were built and trained using a subset of 470 records with and without pre-processing using principal components analysis (PCA). These were subsequently tested on another subset of 417 records. Average sensitivity of $80.75 \%$ (95\% CI $79.54 \%, 81.96 \%)$, specificity of $41.64 \%$ (95\% CI $40.13 \%, 43.15 \%)$, PPV of $46.73 \%(95 \%$ CI $45.20 \%, 48.26 \%)$ and NPV of $77.39 \%(95 \%$ CI $76.11 \%, 78.67 \%$ ) were achieved with the simple perceptron. When PCA pre-processing was used, the perceptrons had a sensitivity of $1.43 \%(95 \%$ CI $1.06 \%, 1.80 \%)$, specificity of $98.32 \%$ (95\% CI $97.92 \%, 98.72 \%)$, PPV of $32.95 \%$ (95\% CI $31.51 \%, 34.39 \%)$ and NPV of $61.33 \%(95 \%$ CI $59.84 \%, 62.82 \%)$. These results show that it is possible for a simple neural network to have respectable sensitivity and specificity levels for angina. (JUMMEC 2006; 9(1): 39-43)
\end{abstract}

KEYWORDS: angina, diagnosis, prediction, decision-support, neural networks, perceptrons

\section{Introduction}

Acute chest pain in the adult is a frequently encountered symptom in all healthcare settings (I). The diagnosis of angina is occasionally not as straightforward as it would appear and in many cases, classical electrocardiogram findings (ECG) of angina may not be evident early on. However, there is broad consensus that lifestyle factors, including physical activity and diet, are fundamental determinants of heart disease risk (2).

In recent years, there has been renewed interest in neural networks in cardiovascular diseases, particularly, ischaemic heart disease. The majority of this research has concentrated on the use of neural networks in diagnosing acute myocardial infraction (AMI) and almost all have used biochemical markers and ECG findings in concert with the history and physical examination. Ellenius and Groth $(3,4)$ investigated neural networks in the assessment of biochemical markers in AMI. Work conducted (5) and in the Heart Disease Program (6) have contributed much to the use of artificial intelligence (Al) techniques in chest pain diagnosis.

Studies have shown very good results with neural networks in the diagnosis of AMI using just patientreportable factors and comparing favourably with statistical methods (7). High sensitivity and specificity levels (exceeding 80\%) for cardiac ischaemia (a mixture of AMI and angina) were also achieved with feedforward neural networks on chest pain patients with ECG and chemical cardiac markers $(8,9)$ and exceeding $90 \%$ (I0) used neural networks to identify patients with significant coronary artery disease and reported positive predictive accuracy rates of $80 \%$ and negative predictive accuracy of $92 \%$ (I I). However, little research has been made using neural networks in diagnosing angina although angina is probably more prevalent than AMI in most developed countries. Furthermore, attempts to build neural networks without the use of any ECG and biochemical markers have been made (12).

Due to the difficulty in diagnosing chest pain and the importance of not missing an important disease like angina where biochemical markers and ECG findings

\section{Correspondence:}

Assoc. Prof. Dr. Awang Bulgiba Mahmud

Department of Social and Preventive Medicine

Faculty of Medicine, University of Malaya

50603 Kuala Lumpur, Malaysia

Tel: 603-7967 4930

Fax: 603-7967 4975

Email: awang@um.edu.my 
may not be as helpful as AMI, this has become an area where some form of decision-support with artificial intelligence (Al) would be most helpful. It would thus be possible to construct a tool or software to aid a paramedic or even the public in diagnosing angina even without the benefit of an ECG with a high degree of probability (possibly exceeding $80 \%$ ).

The objective of this study was to assess the sensitivity, specificity, positive and negative predictive values of simple (single-layer) perceptrons in chest pain diagnosis with respect to the diagnosis of angina. It is also aimed at how far one could use perceptrons in a screening tool that could be used by paramedics or even the public in diagnosing angina.

\section{Materials and Methods}

\section{Source of data}

The data set was obtained from Selayang Hospital, a tertiary level hospital in Malaysia. Permission to use this data for this study was obtained from the Ministry of Health, Malaysia. All records of adult patients (I8 years or older) seen in the Emergency Department for non-traumatic chest pain from 20 August 1999 (when the hospital opened) to 9 August 2002 and clerked using the chest pain clerking form were selected for this study. As this form was used for chest pain suspected to be of cardiac origin, the number of patients clerked using this form was low and the proportion of angina patients clerked using this form was high. Both stable and unstable angina were included as angina as the diagnosis was not clearly stated in many cases. The diagnosis on discharge was used as the definitive diagnosis.

\section{Data cleaning and pre-processing}

Data cleaning and pre-processing were performed before the neural networks were constructed. This involved accuracy checking, treatment of missing values, recategorization and recoding of fields and feature construction. The data was split into two sets; a training set and a test set by random sampling. The training set comprised 470 records while the test set contained 417 records. The training set had 187 cases of angina and the test set, I6I cases of angina. Table I shows the input variables in the data set.

\section{Experimental methods}

The single neuron, single-layer perceptron was used for all experiments. The diagnosis on discharge was used as the definitive diagnosis. This is the diagnosis as confirmed by specialist physicians after taking into consideration ECG readings and other laboratory investigations.

All perceptrons were trained over a maximum of I,000 epochs for each combination of input and target. The perceptrons were then tested on the test set. This process was repeated until ten perceptrons had been trained and tested. Weights were initialized randomly and reinitialized every time the network was trained, so no two networks had the same weights. Matlab 6.5 was used to build, train and test the per-

Table I. Input variables

\begin{tabular}{ll}
\hline Group & Fields \\
\hline Demographic & Age, citizen, race, sex, marital status \\
Nature of chest pain & Location, onset, pattern, quality \\
Radiation of pain & Jaw, left arm, laterally, neck, locally, other parts \\
Relieving factors & Leaning forward, sitting up, GTN, rest, other means \\
Aggravating factors & Posture, meals, coughing, inspiration, exertion \\
Associated heart/lung symptoms & Cough, dyspnoea, oedema, orthopnoea, palpitations \\
Other associated symptoms & Collapse, headache, dizziness, fever, numbness, nausea, sweating, \\
& vomiting, fainting \\
Cardiac risk factors & Age $>40$, diabetes mellitus, family history, hypertension, physical inactivity, \\
& obesity, smoking, known case defaulted treatment, known case on \\
treatment, high cholesterol levels \\
General examination & Pulses, pulse rate, respiratory rate, systolic BP, diastolic BP \\
Heart/lung examination factors & Air entry, breath sounds, chest expansion, chest wall, crepitations, \\
heart sounds, JVP, percussion, pleural rub, praecordium, rhonchi
\end{tabular}


ceptrons. Only patient-reportable factors and examination factors were used in this study as the aim was to investigate, how well the perceptron would be able to diagnose the disease without any investigation results to rely on (Table I).

After using the original data to train the perceptrons, principal components analysis (PCA) was used to reduce the dimensions of the input vectors. The data was thus pre-processed and the perceptrons were retrained and retested. Only input data that contributed to more than $0.5 \%$ of the total variation in the data set were retained.

The results were pooled for each group by the type of pre-processing (10 perceptrons for each group) and outcome measures with $95 \%$ confidence intervals were calculated.

\section{Statistical analysis}

Sensitivity and specificity (13), as well as positive and negative predictive values (14) with $95 \%$ confidence intervals were calculated using standard formulas. The use of a large sample size by pooling the results, is not new (9) and was made in order to obtain a more representative picture of the perceptron performance. This also avoids the problem of confidence intervals exceeding $100 \%$ due to small sample sizes (I5). Continuity correction was used for all calculations of sensitivity, specificity, positive and negative predictive values.

\section{Results}

The performance of the angina perceptron is displayed in Table 2. The training set had 187 cases of angina while the test set had 161 cases of angina. There was no significant difference in training time using the original data and data that had been pre-processed using PCA over 1000 epochs. There was a significant difference in the mean MSE of the two groups with the original data having slightly higher average MSE (0.42I; $95 \% \mathrm{Cl} 0.402,0.439)$ compared to the PCA group $(0.368 ; 95 \% \mathrm{Cl} 0.354,0.382)$ but neither of the MSEs reduced to zero.
Perceptrons trained using the original data managed a respectable mean sensitivity level of $80.75 \%(95 \% \mathrm{Cl}$ $79.54 \%, 81.96 \%)$. This contrast significantly with the perceptrons trained using PCA-processed data, which only managed a dismally low sensitivity of $1.43 \%$ (95\% Cl $1.06 \%$, I.80\%). The difference between these two groups was not only statistically significant but also clinically significant. In contrast, the specificity levels were reversed between the two groups, with the perceptrons trained using PCA-processed data managing a very high specificity level of $98.32 \%(95 \% \mathrm{Cl} 97.92 \%$, $98.72 \%$ ) compared to the low specificity of the perceptrons trained using the original data (41.64\%; $95 \%$ $\mathrm{Cl} 40.13 \%, 43.15 \%)$. Despite the difference in the sensitivity and specificity results, the PPV and NPV levels were significantly better for perceptrons trained using the original data. The PPV for the perceptrons trained on the original data was $46.73 \%(95 \% \mathrm{Cl}$ $45.20 \%, 48.26 \%$ ) while the PPV for the PCA group was $32.95 \%$ (95\% Cl 31.51\%, 34.39\%). NPV for the perceptrons trained on the original data was $77.39 \%$ (95\% $\mathrm{Cl} 76.11 \%, 78.67 \%$ ) while the NPV for the PCA group was $61.33 \%$ (59.84\%, 62.82\%). In general, the variability in test accuracy, sensitivity and specificity levels were much less for perceptrons trained on PCA-processed data compared to those trained on the original data as reflected by their much tighter confidence intervals.

\section{Limitations}

There are a few limitations to these experiments. The single-layer perceptron is limited in what it can do and while it is ideal for problems that have a binary output like a diagnosis, it has some limitations in the amount of information it can conceivably process. These perceptrons have not been tested on a real-time basis but there is no reason to think that it might not work given that it worked on the 417 records (test set) that it had never seen before. This study also did not evaluate all potential patients with ischaemia because it was confined to patients with chest pain in the Emergency Department and thus did not deal with those suffering from silent ischaemia. Finally, this study was carried out at a single institution and one may need to corroborate with patients from different locations.

Table 2. Angina perceptron performance

\begin{tabular}{lcc}
\hline Performance measure & Mean without PCA $(95 \% \mathrm{Cl})$ & Mean with PCA $(95 \% \mathrm{Cl})$ \\
\hline Sensitivity (\%) & $80.75(79.54,81.96)$ & $1.43(1.06,1.80)$ \\
Specificity (\%) & $41.64(40.13,43.15)$ & $98.32(97.92,98.72)$ \\
PPV (\%) & $46.73(45.20,48.26)$ & $32.95(31.51,34.39)$ \\
NPV (\%) & $77.39(76.11,78.67)$ & $61.33(59.84,62.82)$
\end{tabular}




\section{Discussion}

There has been some work with multi-layer perceptrons in chest pain diagnosis and some workers have shown some good results with these $(9,10,16)$. However, most of the work has had to use ECG and biochemical markers and none have used the simple perceptron, perhaps as it is believed that it is not capable of a complex and non-linear process of diagnosing a disease like AMI or angina. Attempts were made for a neural network using just patientreportable factors and without the use of ECG and biochemical markers (I2). It was shown that some uses could be made from the single-layer perceptrons. These perceptrons have the advantage of the hardlimit function and are relatively simple to implement. The advantage is that their relative simplicity makes them less useful where the input data is abstract and they may be unable to "learn" the associations between input and output well enough to be accurate.

The author is encouraged by the sensitivity of 80.75 (95\% Cl 79.54\%, 81.96\%) obtained without PCA processing. Unfortunately, when PCA was used to preprocess the data, it reduced the sensitivity greatly. However, it improved specificity and made the perceptrons consistently more specific compared to the perceptrons trained without PCA pre-processing. One possible explanation for this is that PCA reduces the amount of information needed for the perceptron to make a positive diagnosis and made it unable to differentiate between a positive and negative diagnosis. It would thus tend to label something as being no different from another negative diagnosis. Thus, the perceptron was able to more easily recognize what is not angina rather than what is. Herein probably lies the limitation of the perceptron in pattern recognition. A perceptron can only represent linearly separable functions and are thus not suitable for many functions which are not linearly separable. PCA removes much of the information which is necessary for the perceptron to perform its function properly. However, PCA does have one benefit. It reduces the variability of the perceptron's performance and makes it far more predictable as far as specificity is concerned.

\section{Conclusion}

It seems difficult to come up with a perceptron which is both highly sensitive and highly specific. This is not surprising, as studies have shown similar findings with regards to the balance in sensitivity and specificity (5). Where sensitivity is raised, specificity can be expected to fall as more false positive cases are identified. Research has also shown high sensitivity and specificity levels occurring at the same time (9). To expect the perceptron to achieve sensitivity and specificity greater than 80 per cent at the same time appears to be beyond the single-layer perceptron especially where there are no ECG and biochemical readings to help the perceptrons. In theory, the use of a 2-perceptron system (where one perceptron is trained with PCA and the other is trained without PCA) with the results of both evaluated by a fuzzy inference engine may be able to solve this problem, but it is probably just easier to use a more sophisticated multi-layer neural network instead.

\section{References}

I. Fallon EM, Roques J.Acute chest pain. AACN Clin Issues 1997; 8(3): 383-397.

2. Kelly CNM, Stanner SA. Diet and cardiovascular disease in the UK: are the messages getting across? Proceedings of the Nutrition Society 2003; 62(3): 583-589.

3. Ellenius J, Groth T. Methods for selection of adequate neural network structures with application to early assessment of chest pain patients by biochemical monitoring. Int J Med Inform 2000; 57(2-3): I8I-202.

4. Ellenius J, Groth T. Transferability of neural networkbased decision-support algorithms for early assessment of chest pain patients. Int J Med Inform 2000; 60( I): I-20.

5. Kukar M, Kononenko I, Groselj C, et al. Analyzing and improving the diagnosis of ischaemic heart disease with machine learning. Artif Intell Med 1999; 16(I): 25-50.

6. Long WJ, Fraser H, Naimi S. Reasoning requirements for diagnosis of heart disease. Artif Inell Med 1997; 10(1): 5-24.

7. Wang SJ, Ohno-Machado L, Fraser HSF, et al. Using patient-reportable clinical history factors to predict myocardial infarction. Comput Biol Med 200I; 3I(I): I-I3.

8. Baxt WG, Shofer FS, Sites FD, et al. A neural network aid for the early diagnosis of cardiac ischaemia in patients presenting to the emergency department with chest pain. Ann Emerg Med 2002; 40(6): 575-583.

9. Baxt WG, Shofer FS, Sites FD, et al. A neural computational aid to the diagnosis of acute myocardial infarction. Ann Emerg Med 2002; 39(4): 366-373. 
10. Kennedy RL, Harrison RF, Burton AM, et al. An artificial neural network system for diagnosis of acute myocardial infarction (AMI) in the accident and emergency department: evaluation and comparison with serum myoglobin measurements. Comp Methods Programs Biomed 1997; 52(2): $93-103$.

II. Itchhaporia MD, Snow P, Almassy MS, et al. Artificial Neural Networks: Current Status in Cardiovascular Medicine. J Am Coll Cardiol 1996; 28(2): 515-521.

12. Wang SJ, Ohno-Machado L, Fraser HSF, et al. Using patient-reportable clinical history factors to predict myocardial infarction. Comp Biol Med 200 I; 3I(I): I-I3.
13. Altman DG, Bland JM. Statistics Notes: Diagnostic tests I: sensitivity and specificity. BMJ 1994; 308(6943): I552.

14. Altman DG, Bland JM. Statistics Notes: Diagnostic tests 2: predictive values. BMJ 1994; 309(6947): 102.

15. Deeks JJ, Altman DG. Sensitivity and specificity and their confidence intervals cannot exceed 100\%. BMJ 1999; 318(7177): 193b.

16. Baxt WG, Skora J. Prospective validation of artificial neural network trained to identify acute myocardial infarction. Lancet 1996; 347(8993): 12-15. 